Recibido: 14/05/2019 --- Aceptado: 02/07/2019 --- Publicado: 15/03/2020

\title{
PROPUESTA DE INTERVENCIÓN IMPULSADA POR LA DELEGACIÓN DEL GOBIERNO DE ESPAÑA PARA LA VIOLENCIA DE GÉNERO. ANÁLISIS DE UN DOCUMENTO DE REFERENCIA PARA PROFESIONALES
}

\section{PROPOSAL OF INTERVENTION PROMOTED BY THE GOVERNMENT DELEGATION OF SPAIN FOR GENDER BASED VIOLENCE. ANALYSIS OF A REFERENT DOCUMENT FOR PROFESSIONALS} España. e.aparicio@ucm.es

\section{RESUMEN}

Este trabajo ha llevado a cabo el análisis de una propuesta de pautas de intervención con mujeres víctimas de violencia de género, impulsada por la Delegación del Gobierno de España para la Violencia de Género y dirigida a profesionales. Desde un análisis crítico del discurso, el trabajo tiene como objetivo visibilizar el marco revictimizante desde el que se aborda la intervención ante la violencia de género. También pretende evidenciar una falta de rigor extendida hasta procesos clave que conforman la que debiera ser una atención profesional y que, inevitablemente, impacta en las personas susceptibles de necesitar dicha atención. Consecuentemente, se puede constatar la concepción simplista y reduccionista que subyace de la Intervención Social y que devalúa tanto a ésta como a sus profesionales (mujeres en su mayor parte). Entendiendo el documento como una producción discursiva institucional, sirve como ejemplo del estado de la cuestión. Este trabajo señala la necesidad de mejora de la intervención ante la violencia de género y también algunos de los mecanismos invisibles que sostienen el carácter estructural y sistémico de la desigualdad, tales como la individualización de lo social o la perspectiva de desvalorización científico-profesional de la Intervención Social y, con ella, de las disciplinas implicadas.

PALABRAS CLAVE: intervención social - violencia de género - práctica profesional - análisis del discurso - paradigma sociocrítico - vulnerabilidad - desigualdad.

\footnotetext{
${ }^{1}$ Encarnación Aparicio Martín. Universidad Complutense de Madrid. Pedagoga social y maestra, especialista en Detección e Intervención en Violencia de Género y experta en Igualdad de Oportunidades, doctoranda y docente colaboradora en la Universidad Complutense de Madrid. e.aparicio@ucm.es
} 
Aparicio Martín, E. Propuesta de intervención impulsada por la delegación del gobierno de España para la violencia de género. Análisis de un documento de referencia para

profesionales

\section{ABSTRACT}

This paper has carried out the analysis of a Guidelines Proposal for Intervention with Women Victims of Gender Based Violence promoted by the Government Delegation of Spain for Gender Based Violence and aimed at professionals. From a Critical Discourse Analysis, the work has as objective to visualize the revictimizing framework from which the intervention against gender based violence is addressed. It also aims to evidence a lack of rigour extended to key processes that constitute what should be a profesional care and that inevitably impacts on the sensitive people who may need such care. Consequently, it can be seen the simplistic and reductionist conception that underlies the Social Intervention, and that devalues both the latter and its professionals (women mostly). Understanding this document as an institutional discursive production, it serves as an example of the current state of the issue. This paper points out the neccesity to improve the Intervention against Gender Based Violence, and also some of those invisible mechanisms that sustain the structural and systemic character of inequality, such as the individualization of the social issues or the perspective of scientific-professional devaluation of Social Intervention and, with that, of the involved disciplines.

KEY WORDS: social intervention - violence of gender - professional practice discourse analysis - socio-critical paradigm - vulnerability - inequality.

\section{PROPOSTA DE INTERVENÇÃO PROMOVIDA PELA DELEGAÇÃO DO GOVERNO DA ESPANHA POR VIOLÊNCIA DE GÊNERO. ANÁLISE DE UM DOCUMENTO DE REFERÊNCIA PARA PROFISSIONAIS}

\section{RESUME}

Este trabalho realizou a análise de uma proposta de diretrizes para intervenção com mulheres vítimas de violência de gênero, promovida pela Delegação do Governo da Espanha para a Violência de Gênero e destinada a profissionais. A partir de uma análise crítica do discurso, o objetivo do trabalho é tornar visível o quadro revitimizador a partir do qual a intervenção contra a violência de gênero é abordada. Ele também tem como objetivo demonstrar uma falta de rigor estendida aos principais processos que compõem o que deveria ser cuidado profissional e que inevitavelmente afeta as pessoas que precisam desse cuidado. Conseqüentemente, pode-se verificar a concepção simplista e reducionista subjacente à Intervenção Social e que desvaloriza tanto ela quanto seus profissionais (mulheres na maioria). Entendendo o documento como uma produção discursiva institucional, ele serve como um exemplo do estado das coisas. Este trabalho aponta a necessidade de melhorar a intervenção contra a violência de gênero e também alguns dos mecanismos invisíveis que sustentam a natureza estrutural e sistêmica da desigualdade, como a individualização do social ou a perspectiva da desvalorização científico-profissional da Intervenção Social e, com ela, as disciplinas envolvidas.

PALAVRAS CHAVE: intervenção social - violência de gênero - prática profissional análises do discurso - paradigma sócio-crítico - vulnerabilidade - desigualdade. 
Aparicio Martín, E. Propuesta de intervención impulsada por la delegación del gobierno de España para la violencia de género. Análisis de un documento de referencia para

profesionales

\section{Cómo citar el artículo:}

Aparicio Martín, E. (2020). Propuesta de intervención impulsada por la delegación del gobierno de España para la violencia de género. Análisis de un documento de referencia para profesionales. [Proposal of intervention promoted by the government delegation of Spain for gender based violence. Anlysis of a referent document for professionals]. Revista de Comunicación de la SEECI, 51, 63-82.

doi: http://doi.org/10.15198/seeci.2020.51.63-82

Recuperado de http://www.seeci.net/revista/index.php/seeci/article/view/594

\section{INTRODUCCIÓN}

Esta propuesta, como recoge el propio documento, fue "impulsada" por la Delegación del Gobierno anterior para la Violencia de Género (de la Secretaría de Estado de Servicios Sociales e Igualdad del Ministerio de Sanidad, Servicios Sociales e Igualdad) y sigue en vigor desde la Delegación del Gobierno actual para la Violencia de Género (de la Secretaría de Estado e Igualdad del Ministerio de La Presidencia, Relaciones con las Cortes e Igualdad) ${ }^{2}$. A pesar de no haber actualizado la titularidad del documento, éste se encuentra accesible y definido como "protocolo de actuación" alojado dentro de la "asistencia social integral" en la sección dirigida a "profesionales" y que se describe como "sección dedicada a aportar información y materiales exhaustivos a los diferentes ámbitos profesionales implicados en la violencia de género" ${ }^{3}$.

Que este documento se haya presentado, y aún se presente, de manera institucional, como un protocolo de actuación profesional para ofrecer una atención integral a las víctimas-supervivientes de la violencia de género, le otorga relevancia y le hace susceptible de análisis. Éste, además, podría ser entendido como una herramienta metodológica, transdisciplinar, de carácter ético-político que, por todo ello, podría tener, y de hecho tiene, un alto impacto sobre la vida de las personas.

\section{OBJETIVOS}

Este trabajo, en un sentido amplio, trata de contribuir a la toma de conciencia de la dimensión política de la Intervención Social y, por tanto, de la responsabilidad que diversas profesiones implicadas asumen desde su práctica. El estudio también pretende mostrar cómo el carácter científico-profesional de la Intervención Social puede quedar velado; el reduccionismo al que ésta queda sometida y, por tanto, el

${ }^{2}$ El único dato que se ha logrado conocer sobre edición y fecha de publicación es el año que se menciona en el hipervínculo del mismo nombre que da acceso al documento: "Propuesta de pautas para la intervención integral e individualizada con mujeres víctimas de violencia de género, sus hijos e hijas y otras personas a su cargo (2014).pdf". Se ubicaba en la web oficial de la Delegación para la Violencia de Género del Gobierno en ese momento, y sigue estando accesible en la web oficial de la Delegación del Gobierno actual.

${ }^{3}$ Ver:

http://www.violenciagenero.igualdad.mpr.gob.es/profesionalesInvestigacion/asistenciaSocial/protocolo s/home.htm [Fecha de consulta: 30 de abril de 2019]. 
Aparicio Martín, E. Propuesta de intervención impulsada por la delegación del gobierno de España para la violencia de género. Análisis de un documento de referencia para

profesionales

perjuicio que esto puede suponer, no sólo para las personas y grupos implicados en este ámbito sino para la sociedad en general.

\section{METODOLOGÍA}

Este artículo, a modo de diálogo crítico con el documento estudiado, y desde una perspectiva interpretativa, va presentando interrogantes y señalando elementos para la reflexión. Siguiendo los fundamentos del Análisis Crítico del Discurso recogidos por Martín Rojo (2003,) podría decirse que este trabajo expone algunos elementos de la práctica textual, la práctica discursiva y la práctica social que se desprenden del documento. El foco de atención no sólo se pone en la organización y formato del contenido informativo, sino también en las formas de construcción de representaciones determinadas (estatus, condición, función, relación, etc. de/entre distintos agentes y procesos) desde la incorporación implícita de concepciones, actitudes y valores.

En cuanto a la estructura del trabajo, el artículo consta de dos apartados: el primero presenta la perspectiva de análisis, y se dedica íntegramente a rescatar, desde un paradigma sociocrítico, centros de interés, especialmente sobre la Intervención Social, recogiendo el pensamiento de expertas/os en distintas disciplinas; el segundo, aun incluyendo también alguna referencia al pensamiento de expertas/os, se enfoca en el análisis del documento, tratando de mantener en lo posible la estructura de éste y la nomenclatura de sus apartados, puesto que también éstos quedan sujetos a comentario.

\section{DISCUSIÓN}

\subsection{Perspectiva de análisis}

Desde la Intervención Social como acción práctica, orientada a la resolución de problemas sociales, ésta "es conceptualizada como una forma de actividad que integra aspectos políticos, filosóficos (...). Este hacer está vinculado con saberes teóricos y técnicos, pero especialmente con actitudes, valores y creencias que anteponen eticidad a la acción" (Saavedra, 2015, p. 137). Sin embargo, la intervención no viene definida por la demanda; no escapa al marco histórico, simbólico, conceptual, social de quien interviene, y que es el que termina caracterizando -si no determinando- el tipo de intervención que se lleva a cabo. La intervención nunca podrá ser aséptica; no deja de ser la apuesta política, y el diseño, que los organismos financiadores e interventores hacen del orden social (Cuenca, Sánchez y Burbano, 2014).

Esto contribuye a una doble confluencia de corrientes contrapuestas. Por un lado, llevando la perspectiva de Epstein (Zamanillo, 2012) al contexto español actual, existe, intrínsecamente, una especie de disonancia -si no incongruencia- puesto que abierta y mayoritariamente se actúa para conseguir que las personas se adapten, se acomoden al orden social establecido mientras que, a la vez, supuestamente, se pretende activar la toma de decisiones, el pensamiento creativo, crítico, incluso en 
Aparicio Martín, E. Propuesta de intervención impulsada por la delegación del gobierno de España para la violencia de género. Análisis de un documento de referencia para

profesionales

ciertos ámbitos (como en el que se centra este trabajo) radicalmente desafiante hacia los modelos hegemónicos: un pensamiento que permita la transformación. Por otro lado, se considera que, extrínsecamente, surge un tipo de incongruencia que queda visibilizada a modo de petición de principio, exigiendo, por ejemplo, como requisito previo para el acceso a un servicio, o inicio o continuidad de un proceso, aquello que justamente habría de lograrse desde/a partir de dicho servicio o proceso, conformador del propio proceso de intervención.

En este contexto es imprescindible, además, recordar que un proceso definido como protección, acompañamiento, ayuda, inclusión, cuidado, empoderamiento, etcétera, y que éste venga siendo realizado desde la administración pública o desde la esfera de la solidaridad, no lo exime, en modo alguno, de ser rigurosamente evaluado, no puede quedar exento de análisis ni científico ni ético. Debe combatirse la amenaza de "adiaforización" (utilizando el concepto de Bauman), el aparente esfuerzo permanente por:

situar, a propósito, o por defecto, ciertos actos y/o actos omitidos respecto a ciertas categorías de seres humanos (...) al margen del ámbito de los fenómenos sujetos a evaluación moral; estratagemas para declarar esos actos o esa inacción, de una forma implícita o explícita, como «moralmente neutros» y evitar que las opciones entre ellos se sometan a un juicio ético (...). (Bauman y Donskis, 2015, p. 57)

Por otra parte, cuando se exigen respuestas individuales para afrontar y resolver problemas creados socialmente (Beck en Bauman, 2009), es a las personas a quienes se está culpando exclusivamente por aquellas dificultades que están afrontando (Bauman, 2009; Giroux, 2015). Así quedan entonces sometidas al juicio sistemático acerca de su valía: sus recursos, sus habilidades, la autosuficiencia y el autodominio. Las/os profesionales están sosteniendo esta perspectiva, entre otras razones, porque están siendo instruidas/os desde/para ella. Zamanillo y Martín exponen la falta de "pensamiento crítico global" entre profesionales e intelectuales, y denuncian cómo en el que debiera ser un "proyecto ético-político", sin embargo "la deriva hacia las intervenciones parciales -individuales y familiares- ha copado la formación [en los estudios de Trabajo Social]. El silencio sobre los factores estructurales que producen y reproducen la inequidad, la pobreza y la exclusión social es alarmante" (2011, pp. 102-103).

En cuanto al abordaje de la violencia de género, tan sólo destacar en este apartado que la Declaración de las Defensorías del Pueblo emitida el pasado octubre (2018, p. 2) considera en su punto cuarto que: "los centros especializados (...) requieren de mejoras en el funcionamiento y diseño(...)"; desde el punto quinto se recomienda que: "se refuerce la capacitación y sensibilización, en materia de violencia de género, de todo el personal que interviene(....) mediante formación especializada, continuada, obligatoria y evaluable (...) ", y en su punto octavo sugiere: "la mejora de la calidad de los servicios públicos de atención integral (...), así como la incorporación de técnicas de evaluación de las políticas públicas, elaborando y ejecutando para ello un plan específico de inspección anual." 
Aparicio Martín, E. Propuesta de intervención impulsada por la delegación del gobierno de España para la violencia de género. Análisis de un documento de referencia para

profesionales

Respecto a la relación entre discurso y sociedad, recordar -siguiendo las ideas de Van Dijk (2002)- que el discurso construye, define, cambia las estructuras sociales; las estructuras de éste hablan de o representan partes de la sociedad. Su papel es crucial en las cogniciones sociales. "El discurso tiene implicaciones ideológicas. (...) El discurso es una forma de acción social". (Fairclough y Wodak en Van Dijk, 2016, p. 205). En la práctica de la Intervención Social esto se traduce en asimetrías y, como explica Martín Rojo, en beneficio o detrimento de los intereses de ciertas personas, grupos, comunidades, porque el discurso permite crear "una representación y no otra de los acontecimientos, y ésta a su vez reforzará o cuestionará, naturalizará u objetará unas visiones de los acontecimientos y del orden social y no otras, unas ideologías y no otras" (2003, p. 164).

Por otra parte, Zamanillo pone de manifiesto que en las llamadas profesiones de ayuda se ha renunciado al análisis de los espacios de relación, y la razón principal se encuentra, según la experiencia de esta autora, en:

la tan evidenciada negación de la teoría como intermediación con la práctica. (...) el desdén a la teoría imposibilita reconocer la potente capacidad que tienen las categorías analíticas para nombrar de otra manera los hechos, las relaciones, las contingencias y los fenómenos que nos envuelven y a los que debemos hacer frente. $Y$ nombrarlos de otra forma es poder mirarlos de distinta manera, mirada que nos ayudará a intervenir diferentemente (2012, pp. 158-159).

Por ello, entrelazar práctica y teoría es la única vía efectiva para lograr una cierta amplitud en la mirada que permita tomar conciencia sobre la complejidad no sólo de la violencia de género u otros problemas sociales, sino de la Intervención Social ante ellos, o -al menos- de la temeraria, perniciosa, simplificación de ésta.

\subsection{Ejercicio de análisis}

"Propuesta de pautas para la intervención integral e individualizada con mujeres víctimas de violencia de género, sus hijos e hijas y otras personas a su cargo" 4

\section{Marco de Referencia}

La premisa de la que se parte es que éste es un documento de referencia dirigido a las/os profesionales puesto que, como se indica en el cuarto párrafo de este apartado, la Delegación del Gobierno para la Violencia de Género: "impulsa esta propuesta que contiene pautas y elementos mínimos que podrían tenerse en cuenta en la intervención integral dirigida a mujeres víctimas de violencia de género", y que en el siguiente párrafo se presenta como: "marco de referencia orientativo para

\footnotetext{
${ }^{4}$ En el intento de respetar la estructura del documento estudiado, se mantiene -en la medida de lo posible- la nomenclatura y el orden de las partes analizadas. No se conserva el formato de mayúsculas en los distintos epígrafes ni al reproducir partes del texto para facilitar la lectura y no inducir a error respecto a la estructura de este artículo.
} 
Aparicio Martín, E. Propuesta de intervención impulsada por la delegación del gobierno de España para la violencia de género. Análisis de un documento de referencia para

profesionales

todos los profesionales que con formación y supervisión específica, desempeñan su trabajo en las distintas redes de recursos especializados, residenciales o no residenciales (...) para mujeres víctimas de violencia de género y sus hijos/as".

¿Propuesta de pautas? ¿Pautas y elementos? Pautas y elementos "mínimos" que "podrían tenerse en cuenta": Es decir, no necesariamente. ¿De cuáles se puede prescindir? ¿Son mínimos, pero no es necesario tenerlos en cuenta? Marco de "referencia orientativo": De referencia. Pero orientativo. "Profesionales con formación": ¿Profesionales de qué? ¿Con formación? ¿Qué formación? ¿Es que puede darse la circunstancia de que la intervención sea desarrollada por personal no cualificado? "Supervisión específica": ¿Supervisión? ¿Específica? ¿Quién o quiénes supervisan? ¿A quién interpela este documento? ¿Tienen otro documento exclusivamente dirigido a ellas/os quienes supervisan?

Este conjunto de interrogantes ya deja ver que, desde el formato y la redacción hasta la terminología y la secuenciación de contenido, todo ello va a disponer inevitablemente hacia un contexto de acción informal, laxa, ambigua, inconsistente. Por otra parte, el documento no contempla como necesario el lenguaje inclusivo puesto que el mismo texto no lo aplica. Utiliza el masculino genérico en varias ocasiones al referirse, por ejemplo, a "todos los profesionales" y más tarde a "un profesional de referencia", a pesar de tratarse de un trabajo dirigido al abordaje de la violencia de género (máximo exponente ésta de desigualdad) y de que la inmensa mayoría de las/os profesionales que trabajan con mujeres víctimas-supervivientes de violencia de género son mujeres. También se puede leer "del menor", "los menores" o "hijos/as" a pesar de que la a es anterior a la oy pudiera escribirse hijas/os.

Se alude al Catálogo de Referencia de Servicios Sociales y a los criterios comunes de calidad y de "buen uso -solidario y responsable- de los servicios" en el segundo párrafo de la primera página del documento ${ }^{5}$. El uso y posición de este mensaje en el texto denota una priorización que revela los prejuicios que subyacen. Más allá de la revictimización que podría suponer que los derechos quedaran reconvertidos en privilegios, resulta obligado recordar que se pretende -como el propio documento reconoce- una atención "integral" que en muchas ocasiones exige una cobertura de necesidades básicas para garantizar la protección, o incluso la intervención en crisis, entre otras muchas actuaciones.

Más allá de las frecuentes lesiones que trae consigo la violencia física e incluso discapacidades como sordera o pérdida de visión, y otros tantos graves daños que tienen la violencia en su base, como fibromialgia y colon irritable, no pueden olvidarse aquellos problemas que aun calificados como de menor gravedad (gastrointestinales, cefaleas, dolores musculoesqueléticos, entre otros) traen consigo, sin duda, un importante deterioro de la calidad de vida de las mujeres que los sufren. También son frecuentes los daños en la salud sexual y reproductiva (ETS,

${ }^{5}$ El número de página viene establecido por la autora de este artículo puesto que el documento publicado no está paginado ni cuenta con índice alguno. 
Aparicio Martín, E. Propuesta de intervención impulsada por la delegación del gobierno de España para la violencia de género. Análisis de un documento de referencia para

profesionales

disfunciones sexuales, problemas ginecológicos y en el embarazo) y, en un importante porcentaje, están presentes los intentos autolíticos y la ideación suicida. Por otra parte, las consecuencias de la violencia se cronifican y trascienden al maltrato; las vivencias traumáticas traen una profunda vulnerabilidad, sufrimiento (Mateo y Bueno, 2018, pp. 57-59). Entre las consecuencias psicosociales, pueden destacarse ansiedad, depresión y baja autoestima, trastorno de estrés postraumático, déficit de atención y concentración, hiperactividad, inhibición y aislamiento, comportamientos de riesgo, dificultades de aprendizaje y de socialización y violencia transgeneracional con alta tolerancia a situaciones de violencia, entre otros (Aretio, 2018, p. 75). Es decir, se está muy lejos de presentar, por ejemplo, un programa de turismo (sin pretender restar todo el respeto que éste merece).

En el cuarto párrafo se señala que "con el fin de promover instrumentos que garanticen la suficiencia y calidad de la intervención personalizada y multidisciplinar (...) impulsa esta propuesta". Sin embargo, la suficiencia ${ }^{6}$ no es un elemento sumatorio al concepto de calidad; al menos en el contexto que nos ocupa ésta sería del todo inexistente sin aquella. Señalar el pleonasmo utilizado para obviar el significado del concepto calidad y convertir una condición sine qua non en un elemento meramente deseable. Parece que existiera la posibilidad de ofrecer un servicio insuficiente, así que la idea que subyace es que la atención es prescindible. De nuevo, un derecho reconvertido en un privilegio.

La pretensión del documento es "que las mujeres víctimas de violencia de género (...) disfruten de unas condiciones semejantes y con unos criterios comunes en la atención que reciben". Hay que resaltar las connotaciones que tiene el hecho de utilizar el verbo "disfrutar" (en vez de "disponer", por ejemplo) al referirse a las condiciones de atención. Vuelve a resultar revelador que se elija un término que evoca deleite, que construye un imaginario de actividades placenteras.

\section{Fines}

En el apartado "fines", en el primer párrafo, se utiliza la expresión "dándoles la oportunidad de alejarse del foco de la violencia de género", en vez de posibilitar, que probablemente sería más acertado, por poner un ejemplo. Primero se "disfruta de las condiciones", y ahora "se da la oportunidad". Una vez más es un privilegio, no un derecho. En cuanto a alejarse del "foco de violencia": ¿Foco? ¿Es que la violencia es ejercida por un ente?, ¿algo abstracto que no se puede nombrar ni definir?

En este mismo punto, se establece como fin "Garantizar (...) acompañamiento cualitativo": tan sólo decir que no se ha alcanzado a comprender a qué se refiere el documento con esta expresión; tal vez hubiera resultado más fácil si se hubiera logrado encontrar un ejemplo de acompañamiento sólo "cuantitativo".

6 Según el Diccionario de la Real Academia Española (DRAE), "sufficientia", del latín, significa "capacidad", y como locución adverbial, "bastantemente". 
Aparicio Martín, E. Propuesta de intervención impulsada por la delegación del gobierno de España para la violencia de género. Análisis de un documento de referencia para

profesionales

Más allá de un espacio y tiempo para la recuperación "emocional" (parece que sólo es necesaria ésta, y no hubiera más daños que perduren) se especifica concretamente la necesidad de "la toma de conciencia", sin mayor precisión. ¿Qué es la toma de conciencia? Esta falta de precisión invita a pensar que pueda estar asumiéndose la idea de que por el mero hecho de ser víctima-superviviente de la violencia de género se está, sistemáticamente, en una situación de inconsciencia generalizada; de hecho, pocas líneas más adelante el texto plantea como uno de los fines promover "la responsabilidad". ¿A qué profesionales va dirigido este texto, especialistas en qué disciplinas? ¿Qué concepto se tiene de la violencia de género? ¿Son un colectivo las mujeres "víctimas de violencia de género"? Por otra parte, incluso desde la responsabilidad como capacidad para responder, ¿no tiene ésta vínculo alguno con la capacidad de respuesta del entorno?

Es necesario resaltar también el reduccionismo que supone identificar víctimasuperviviente de violencia de género con persona en situación de dificultad socioeconómica; incidir "especialmente" en la "inserción y formación laboral" (con estos términos y no otros) parece dar prueba de ello. Aun sin olvidar que -como ONU Mujeres (2015) explica- las mujeres que han estado expuestas a la violencia corren mayor riesgo de sufrir dificultades ante/en el empleo, falta de vivienda y, en general, aislamiento social y pobreza, es ciertamente pernicioso entender a las mujeres víctimas-supervivientes de violencia de género como colectivo, y atribuirles unas características concretas, obviando así lo que también queda argumentado por el organismo anteriormente citado: que ante el riesgo de sufrir violencia no existen características fijas o inherentes a determinadas personas o grupos; "mujeres de todo el espectro social sufren la violencia y son muchos los factores que contribuyen a su perpetración" (ONU Mujeres, 2015, p. 19).

El último de los fines "en relación a las mujeres" que el documento señala es: "apoyar a las mujeres (...) para que puedan abandonar su posición de víctimas". Esto sugiere, cuanto menos, que el acento se pone en lo actitudinal $y$, por tanto, podría denotar cierto juicio, puesto que se elige posición frente a situación. Además, se albergan grandes dudas acerca del uso del verbo "abandonar" puesto que, teniendo en cuenta el tono general del documento, éste invita a pensar que, de nuevo, se parte de la idea de una corresponsabilidad ante la "posición" inicial (elegida y conservada), algo a lo que ha de renunciarse, que ha de dejarse; ; no sería descabellado pensar que esta expresión de "abandonar su posición de víctimas" no hace sino culpabilizarlas.

Hay que señalar también que, en el documento (al menos en el sexto punto de este apartado) se opta por referirse a las mujeres como "la mujer acogida" a pesar de que el documento no especifica que se trate de una propuesta de pautas de intervención exclusivamente dirigida a los recursos residenciales, o "casas de acogida" (que es la nomenclatura a la que el texto aquí evoca). En cualquier caso,

7 Según el DRAE, las tres primeras acepciones de "abandonar" implican "dejar de". La primera acepción de "posición" es: "postura, actitud o modo en que alguien o algo está puesto". 
Aparicio Martín, E. Propuesta de intervención impulsada por la delegación del gobierno de España para la violencia de género. Análisis de un documento de referencia para

profesionales

incluso en tal contexto residencial, las mujeres (no son) ${ }^{8}$ están en situación de protección.

"En relación a los y las menores y otras personas dependientes de la mujer" (o como se podría hacer referencia a éstas/os ultimas/os: personas en situación de dependencia) el documento insiste en dar "la oportunidad" de alejarse del "foco" de la violencia, y propone en el tercer punto "proporcionar a través de la atención y el abordaje integral de todas sus necesidades (psicológicas, sociales, educativas, jurídicas) un ámbito para crecer y desarrollarse, realizando a la vez las responsabilidades sociales que les corresponde según su edad". Más allá del hecho de que el pensamiento parece seguir puesto en lo residencial, de nuevo no se desaprovecha la ocasión para aludir a las "responsabilidades" "que les corresponde", "según su edad". ¿"Realizando a la vez responsabilidades sociales"? Esta expresión sirve como ejemplo de la redacción, uso de conceptos, etcétera, que se da tanto en este apartado como en el resto del documento.

\section{Admisión al recurso ${ }^{9}$}

\section{Elementos a considerar y objetivos}

Comenzar señalando que el término "admisión" suscita muchas dudas, pero lo que resulta del todo incomprensible es un apartado denominado "elementos a considerar y objetivos". Este es otro ejemplo respecto al nive/ técnico que refleja el documento y, por tanto, la falta de profesionalidad con la que se está abordando este asunto. Se ha de destacar, dentro de este apartado, el texto que, con letras en negrita y mayúsculas dentro de un cuadro coloreado en gris, a modo de alerta o recordatorio, se ubica al final de la página.

ES IMPORTANTE DISPONER EN ESTE MOMENTO DE TODA LA INFORMACIÓN RELEVANTE, RECABADA DE LA USUARIA Y DE OTROS RECUROS PARA EVITAR QUE ELLA Y SUS HIJOS/AS Y OTRAS PERSONAS A SU CARGO TENGAN EN MOMENTOS SUCESIVOS QUE VOLVER A REFERIRSE A INFORMACIONES QUE YA OBREN EN PODER DE LA ADMINISTRACIÓN EVITANDO REVICTIMIZACIONES. ASIMISMO FACILITA QUE LAS ACTUACIONES DE LOS DISTINTOS PROFESIONALES SEAN COHERENTES ENTRE SÍ

Disponer de "toda" la información "relevante" "facilita" que las distintas actuaciones sean "coherentes". Una vez más surge la pregunta: ¿a quién va dirigido este texto?, ¿a profesionales? Tal vez se está dando por hecho que es importante recordar esto. Si es así, o se está asumiendo que no están cualificadas/os, o bien

\footnotetext{
${ }^{8}$ Incluso centrando la mirada en un recurso residencial, y focalizándonos en la situación de "riesgo social" las mujeres no son "mujeres acogidas", en todo caso "están en situación de acogida". De todas formas, el criterio para acceder a un recurso residencial es, en principio, el "riesgo vital", independientemente de que también pudiera darse riesgo social. Por tanto, ha de hablarse de protección, no tanto de acogida.

${ }^{9}$ En este punto del documento, (corresponde a una tercera página) aparece de nuevo el título del documento enmarcado al inicio de la página. Se opta por evitar reproducirlo estimándose que podría inducir a error.
} 
Aparicio Martín, E. Propuesta de intervención impulsada por la delegación del gobierno de España para la violencia de género. Análisis de un documento de referencia para

profesionales

que tal vez no puedan intervenir en/desde las condiciones que la situación requiere. En cualquier caso, parece que ambas situaciones pueden asumirse con total naturalidad puesto que un cuadro gris con texto en mayúsculas será de suficiente ayuda.

En el segundo párrafo se explicita (no se puede deducir si como "elemento a tener en cuenta" o como "objetivo") que ha de informarse a "la mujer" acerca de las "normas de funcionamiento" del recurso más adecuado a sus necesidades; en el cuarto párrafo, se explicita también que ha de facilitarse un documento que explique derechos, "deberes y obligaciones" y "normativa de funcionamiento" del recurso en el que se encuentre. ¿Realmente no hay otros "elementos a considerar y objetivos" que merezcan espacio en un documento como éste, de referencia, y que puedan aportar más a la intervención? ¿Realmente es imprescindible priorizar esto e insistir en informar acerca de deberes, y obligaciones, y normativa?

La redacción del sexto punto merece ser reproducida en su totalidad: "Apoyar a la usuaria y a la unidad familiar en la admisión al recurso creando un clima de confianza y empatía propicio para la creación de vínculo con el equipo del recurso, sin crear expectativas y necesidades no realistas". ¿Apoyar en la admisión? ¿Explicitar "elementos/objetivos" tales como empatía? Se insiste: ¿a quién va dirigido este documento? Por último: ¿"sin crear expectativas y necesidades no realistas"?

En el octavo punto se indica literalmente que se han de "evaluar y recoger" expectativas y necesidades planteadas. ¿Evaluar y recoger? "para posteriormente definir lo objetivos, por áreas que deberán contar en el Plan de Actuación Individualizada (PAI)". "Objetivos por áreas que deberán contar en el plan (...)". Para terminar el apartado, en el último punto se insta a establecer "una fecha para la elaboración y las evaluaciones del Plan de Actuación Individualizada (PAI)". No se distingue entre "elementos", "objetivos", "evaluar", "recoger", pero es importante establecer una fecha.

\section{Evaluación e intervención: Formulación del plan de actuación individualizado (PAI)}

Comenzar señalando que no se logra comprender la estructura establecida: El primer apartado se dedica a la "admisión"; el segundo a "Evaluación e intervención: formulación del PAI". Además, La redacción del texto lleva a pensar que se mantiene la mirada puesta permanentemente en un solo tipo de recurso (residencial), lo que implica aún mayor reduccionismo y evidencia que la desvalorización y desprofesionalización de la intervención son especialmente significativas en este tipo de recursos y contextos.

Se describe el PAI como un "documento", "un documento que debe servir de guía durante toda la intervención". Se explica que "sitúa a mujer y profesionales durante todo el proceso de intervención permitiendo no perder de vista los objetivos definidos con claridad consensuados con la mujer". De nuevo, un ejemplo de la laxitud (por usar un eufemismo) que se presupone podría llegar a darse. Una vez 
Aparicio Martín, E. Propuesta de intervención impulsada por la delegación del gobierno de España para la violencia de género. Análisis de un documento de referencia para

profesionales

más sólo caben dos preguntas: ¿a quién va dirigido este documento? ¿Qué tipo de intervención se asume que podría realizarse o estar realizándose?

Ni en los apartados anteriores ni en éste se dedica espacio alguno a la evaluación. Se menciona de soslayo, por ejemplo, para "evaluar y recoger expectativas y necesidades" y "establecer una fecha para la elaboración y en su caso las evaluaciones periódicas del PAI". También se podría rescatar su vinculación con términos como "valoración", "diagnóstico" y expresiones como "acreditado el cumplimiento inicial de los objetivos", "revisar el cumplimiento del PAI"10 o la mención en las distintas áreas al "seguimiento" del PAI, especificando que su realización "sería deseable" (tampoco necesaria, por tanto) ${ }^{11}$ y se hará "en función del periodo de permanencia de la mujer en el recurso". No hay más mención a proceso evaluativo alguno, ni aisladamente tampoco a ninguno de los criterios que pudieran conformarlo. Respecto a ese deseable seguimiento, en función de la permanencia, ni siquiera se logra entender exactamente cuál es el planteamiento: ¿La intervención en función de la "permanencia" y no la "permanencia" en función del resultado de la intervención?

\section{Elementos a considerar y objetivos}

En el primer punto repentinamente se menciona un Plan de "Atención" Individualizado (entendemos que como sinónimo del anterior Plan de Actuación). Por su parte, las mujeres pasan de ser "mujeres" a "usuarias", o "mujer", o "usuaria". Otro ejemplo de la falta de precisión en la redacción del texto y, por lo tanto, la falta de rigor que presenta todo el documento. En cuanto al resto del apartado, se presenta a modo de lluvia de ideas. Puede definirse como un frangollo que mezcla procesos, niveles, ámbitos, etcétera, y que (aun salvando, lógicamente, ciertos "elementos a considerar y objetivos") deja entrever una perspectiva que trata a las mujeres "víctimas de violencia de género" como un colectivo, parece que comparten características concretas como la tendencia a la irresponsabilidad y a la dependencia.

Si se busca realizar un diagnóstico, "evitando la patologización" ¿por qué se utiliza más tarde el término tratamiento? ¿Así es como se evita la patologización? Por otro lado, no se insta a garantizar las condiciones que faciliten la recuperación emocional, tan sólo "promover la recuperación del daño emocional" y lo más sorprendente: esta "promoción" queda al mismo nivel que el establecimiento de "reuniones de coordinación internas y externas (...) y el establecimiento de "los mecanismos de derivación que se precisen (...)." Reuniones de coordinación "que faciliten a los

\footnotetext{
${ }^{10}$ Estas dos expresiones se encuentran en el tercer apartado "Salida/Alta/Seguimiento", que cuenta con un solo subapartado denominado "Elementos a Considerar y Objetivos".

${ }^{11}$ Que el "seguimiento" del PAI sea "deseable" abre las puertas a una actividad (no se va a definir ni siquiera como atención) que dista mucho de poder ser considerada seria, menos aún profesional, menos aún de calidad. Este es un ejemplo más de cómo este documento contribuye a ofrecer una idea simplista y reduccionista que no hace sino denostar el sector profesional de la "Intervención Social" y con él las disciplinas científicas implicadas en ella y las competencias y actuación de sus profesionales.
} 
Aparicio Martín, E. Propuesta de intervención impulsada por la delegación del gobierno de España para la violencia de género. Análisis de un documento de referencia para

profesionales

profesionales orientar los objetivos establecidos de una forma clara y congruente con la intervención que se quiere realizar y con la misión del recurso": ¿Orientar los objetivos establecidos?, ¿orientar de forma clara y congruente los objetivos con la intervención que se quiere realizar? ¿Orientar los objetivos con la misión del recurso?

Adviértase, también, que en ningún momento parece contemplarse la posibilidad de formación en feminismo, o educación para la igualdad ${ }^{12}$ (sólo algo más sutil, "facilitar conocimientos y habilidades"). Pero lo que podría considerarse grave, sin duda, es la ausencia del término "empoderamiento", especialmente mientras no se duda en trasladar - como mensaje principal coloreado y en mayúsculas- que debería motivarse para "el tratamiento". No caben más comentarios ante el texto coloreado que se destaca como alerta o recordatorio al final del apartado:

ES IMPORTANTE PROPICIAR QUE LA USUARIA DEMANDE LA INTERVENCIÓN MEDIANTE LA TOMA DE CONCIENCIA DE LA NECESIDAD DE UN PROCESO DE RECUPERACIÓN; FOMENTAR EXPECTATIVAS DE FUTURO ORIENTADAS A LA AUTONOMÍA Y LA INDEPENDENCIA Y EVITANDO LA INSTITUCIONALIZACIÓN Y LA DEPENDENCIA DE LA USUARIA. PARA ELLO, UNO DE LOS PRIMEROS ASPECTOS A TRABAJAR DEBERÍA SER LA MOTIVACIÓN PARA EL TRATAMIENTO.

\section{Propuesta de PAI (Plan de Atención Individualizada) ${ }^{13}$}

\begin{tabular}{|c|}
\hline $\begin{array}{c}\text { PROPUESTA DE PAI } \\
\text { (Plan de Atención Individualizada) }\end{array}$ \\
\hline INTRODUCCIÓN - ANTECEDENIES \\
\hline DATOS PERSONALES \\
\hline $\begin{array}{l}\text { Historia de violencia de género y vía de acceso al recurso } \\
\text { Historia de relación: } \\
\text { 1. Tiempo de relación (noviazgo, matrimonio, convivencia) } \\
\text { 2. Inicio del maltrato } \\
\text { 3. Lugar habitual donde se produce el maltrato (domicilio, vía pública, lugar de } \\
\text { trabajo, etc.) } \\
\text { 4. Uso/amenaza con armas } \\
\text { 5. Valoración tipología de maltrato } \\
\text { Tiempo previsto de estancia: (meses) }\end{array}$ \\
\hline Zonas de riesgo para la mujer. Indicar dispositivos de protección. \\
\hline
\end{tabular}

No se va a insistir más en las dificultades que se encuentran para entender la estructura, tampoco expresiones como "Introducción-Antecedentes", o en sí mismo

${ }^{12}$ Se quiere hacer notar que, paradójicamente, el término educativo suele evitarse, probablemente porque se está asumiendo la tan nociva y extendida idea de que éste supone una infantilización, sin embargo, no supone problema alguno asumir el riesgo de psicopatologización o medicalización.

${ }^{13}$ En este momento el PAI vuelve a definirse como Plan de Atención y no de Actuación. La estructura que se presenta resulta tan incomprensible que se opta por incluir la imagen completa del título y el primer apartado. 
Aparicio Martín, E. Propuesta de intervención impulsada por la delegación del gobierno de España para la violencia de género. Análisis de un documento de referencia para

profesionales

todo el apartado de "Historia de relación" (elementos que lo conforman y omisiones). Incidir tan sólo en que se solicita que se especifique el tiempo previsto de estancia "(meses)". Es decir, un documento llamado "Propuesta de Pautas para la Intervención Integral e Individualizada con Mujeres Víctimas de Violencia de Género, sus Hijos e Hijas y otras Personas a su cargo" recoge un "Plan de Actuación/Atención Individualizada" que parte de un tiempo previsto, (y además no de intervención, atención, acompañamiento, asesoramiento, apoyo o participación, etc.): de estancia. ¿En qué recurso específico se está pensando? ¿Qué recursos, intervenciones, informaciones previas se presuponen? ¿Qué profesionales previamente implicadas/os? ¿De dónde se parte, a dónde se quiere llegar? Se podría continuar formulando preguntas ad infinitum.

Después del apartado llamado introducción-antecedentes, se encuentran apartados coincidentes con las áreas: "socio-educativa", de Trabajo Social, jurídica, psicológica (ésta queda dividida en dos subapartados: mujer, y menores y/o personas a su cargo) y, por último, área "educativa infantil". En todas ellas, al inicio se explica que "se muestra la información que se considera importante recoger en el área", "desde los distintos recursos que trabajan con la mujer y sus hijos/as". Y al final de cada área se repite el siguiente texto:

OBJETIVOS: COMPROMISOS, ACTUACIONES, Y SEGUIMIENTO

Los objetivos se plantean a corto plazo ya que sería deseable realizar un seguimiento del PAl en función del periodo de permanencia de la mujer en el recurso.

OBSERVACIONES

Es decir: por una parte, ahora se explica a las/os profesionales la información "que se considera importante recoger" desde "distintos recursos" $y$, por otra parte, se propone la formulación o establecimiento de lo que podría quedar cortésmente definido como maremágnum: "Objetivos: compromisos, actuaciones y seguimiento". La evaluación de la intervención sigue sin tener suerte de encontrar su espacio, ni aleatoriamente. Tampoco se esclarecen los criterios para la selección de "información" y, respecto a las distintas áreas, sólo cabría señalar que, en el mejor de los casos, como el propio documento indica, éste recoge cierta recogida de datos. Desde aquí no cabe la posibilidad de mayor análisis puesto que tan sólo se trata, de nuevo, de grupos de lluvias de ideas, o listados. En este apartado, el contenido, en algunas áreas tales como la "socio-educativa" y la "educativa infantil", destacan por su vacuidad y desestructuración.

El documento efectivamente equipara un "Plan de Atención Individualizada" con una recogida de datos. Sólo recordar a Zamanillo cuando reivindica trascender las listas de hechos o de situaciones-problema en el análisis de la realidad, insistiendo en la necesidad de contar con saberes que permitan conceptualizar: "sin conceptos estamos ciegos porque es el acto de nombrar lo que crea realidades y objetos de investigación, no la mera relación de datos de las realidades que conforman el mundo en el que intervenimos" (2012, p. 166). Hay que destacar, además, lo pernicioso de identificar "objetivo" con "actuación" y "evaluación" con "diagnóstico". Y, por último, insistir en el impacto que supone leer una y otra vez en cada apartado 
Aparicio Martín, E. Propuesta de intervención impulsada por la delegación del gobierno de España para la violencia de género. Análisis de un documento de referencia para

profesionales

correspondiente a las distintas áreas: "Los objetivos se plantean a corto plazo ya que sería deseable realizar un seguimiento del PAI en función del periodo de permanencia de la mujer".

\section{Valoración y conclusiones}

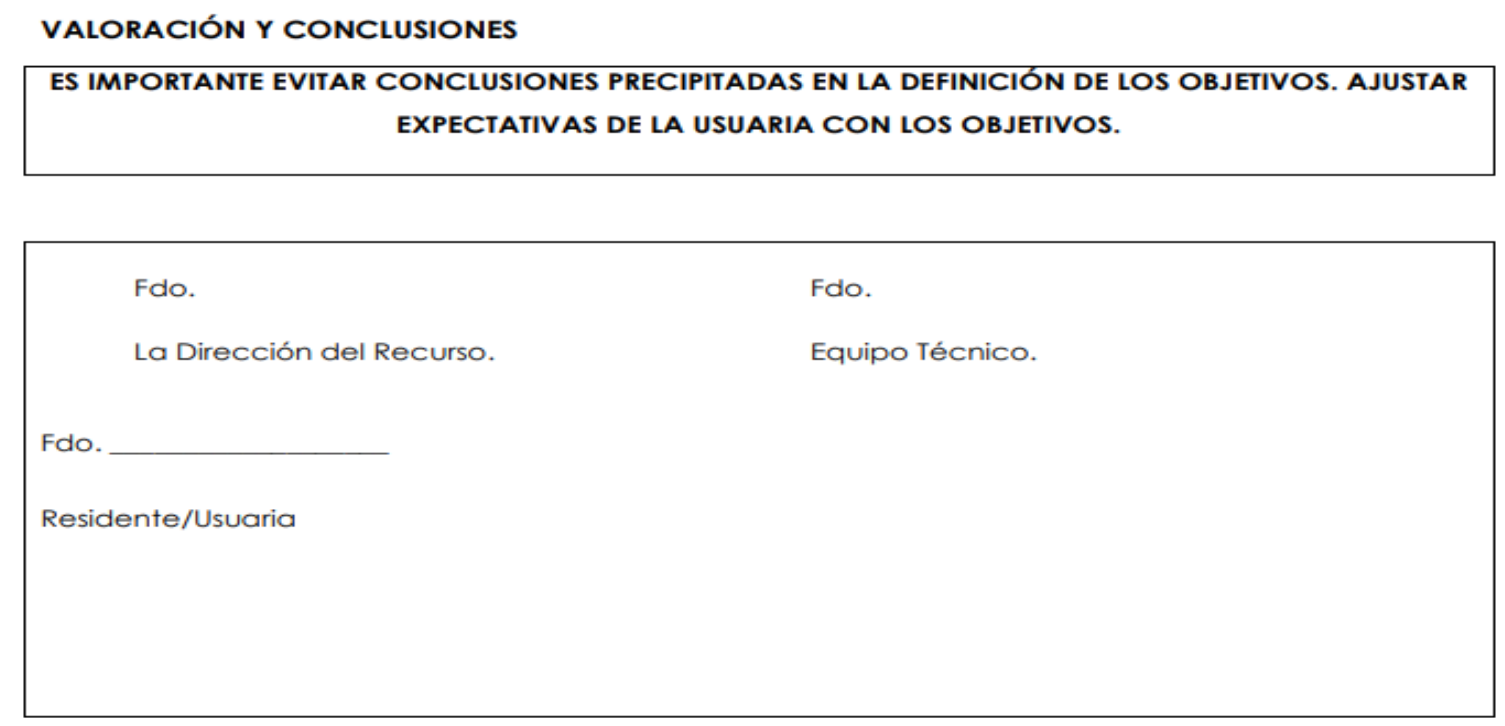

No se puede más que repetir a modo de interrogantes: ¿Valoración y conclusiones? ¿Importante evitar "conclusiones precipitadas"? ¿Evitar "conclusiones precipitadas en la definición de los objetivos"? ¿"Ajustar expectativas de la usuaria con los objetivos"?

Para finalizar el apartado dedicado al plan de atención individualizada se incluye lo que podría entenderse como un ejemplo de cómo debe oficializarse el plan: la firma de la "Dirección del Recurso", del "Equipo Técnico" y, bajo éstas, la firma de la "Residente/Usuaria". Por lo visto, el documento considera imprescindible dedicar un espacio a instruir en la firma del PAI.

\section{Salida/Alta/Seguimiento}

\section{Elementos a considerar y objetivos}

No se alcanza a comprender cómo se puede "concluir el proceso de intervención una vez acreditado el cumplimiento inicial de los objetivos" (según se expone en el primer punto del apartado). ¿Cumplidos inicialmente? A su vez, el segundo punto vuelve a alzarse como ejemplo de calidad técnica: "Revisar el cumplimiento del PAI, valorando objetivos cumplidos y las posibles dificultades". ¿Revisar cumplimiento valorando objetivos cumplidos? Y algo cuanto menos sorprendente: "Capacitar a la mujer superviviente para servir de apoyo y ejemplo en la recuperación de otras mujeres". Destaca, por un lado, el uso de "superviviente" (por primera y única vez en el documento) y, por otro, el propósito explícito de lograr que la mujer sirva de apoyo. Y de ejemplo. 
Aparicio Martín, E. Propuesta de intervención impulsada por la delegación del gobierno de España para la violencia de género. Análisis de un documento de referencia para

profesionales

En general, todo el apartado no deja de ser otra lluvia de ideas, entre el simplismo y la imprecisión; de hecho, se denomina: "Salida/Alta/Seguimiento" y su superficialidad queda reflejada en puntos como los que señalan la necesidad de acordar pautas de seguimiento con "la usuaria" o establecer un sistema de seguimiento para "ver la evolución de la mujer". El mensaje que se destaca (usando el habitual formato de cuadro de texto coloreado en gris y con letras mayúsculas) es el siguiente:

ES IMPORTANTE FACILITAR QUE LA MUJER CUENTE CON LAS CONDICIONES MATERIALES APROPIADAS PARA ELLO, EN LO QUE SE REFIERE A VIVIENDA Y EMPLEO, AYUDA A EVITAR LA INSTITUCIONALIZACIÓN O EN SU CASO, LA DERIVACIÓN A OTRO RECURSO RESIDENCIAL O NO.

De nuevo, la elección del lenguaje denota una perspectiva muy concreta que se vislumbra en el punto de partida (una vez más desde un recurso específico y ante unas necesidades muy específicas) y en lo que se propone al respecto. ¿Qué significa exactamente "condiciones materiales apropiadas" para la salida/alta? ¿Es importante facilitar?, ¿facilitar que cuenten con empleo y vivienda? No es imprescindible, no hay que garantizar; sólo es importante facilitar que ellas... Es decir: permisivo de la exclusión, si no activamente excluyente. $Y$, además, no porque sean derechos sino porque "ayuda a evitar la institucionalización o la derivación a otro recurso". En realidad, ya en el apartado dedicado a la formulación del plan de actuación individualizado, se señalaba, en la nota coloreada, lo importante de "fomentar expectativas de futuro orientadas a la autonomía y la independencia, evitando la institucionalización y la dependencia". Estas ideas, así expresadas, nos retrotraen a la caridad victoriana; refiriéndose a Octavia Hill y al movimiento de "visitadoras amistosas", Walkowitz explica: "Tenían que ofrecer ayuda espiritual y disciplina a «los inquilinos que, por su falta de voluntad, necesiten impulso permanente so pena de quedarse irremisiblemente atrás» (Miranda, 2009, p. 105).

Señalar, además, que ese limitarse a "facilitar" no encuentra correspondencia con el primer punto de este apartado: "Concluir el proceso (...) disponiendo la usuaria de los recursos y apoyos necesarios para su vida autónoma" (sí puede apreciarse que el documento termina como empieza: al inicio nos recuerda que ha de hacerse un uso solidario y responsable de los recursos, y al final insiste en que se evite la derivación a otro recurso).

Puesto que el documento pone de nuevo el foco de atención en el empleo, mencionando ahora, además, el derecho a la vivienda, "facilitar", no garantizar, evidencia, como ya se ha mencionado, no sólo la exigencia de la capacidad de respuesta individual, sino también el permanente ejercicio por ocultar la privación como índice para definir la pobreza ${ }^{14}$, e invisibilizar también el auténtico alcance de

\footnotetext{
${ }^{14}$ Parece que desde esa mención especial que en el documento se hace a la formación y al empleo ya queda cubierto el objetivo de "facilitar" el acceso a la vivienda (sin embargo, en la España actual ni la formación garantiza un empleo, ni un empleo a tiempo completo garantiza la posibilidad de cobertura
}

Revista de Comunicación de la SEECI. 15 marzo, 2020 / 15 julio, 2020, n 51, 63-82 
Aparicio Martín, E. Propuesta de intervención impulsada por la delegación del gobierno de España para la violencia de género. Análisis de un documento de referencia para

profesionales

la exclusión social. Apoyándose en el trabajo de Gordon y otros autores, Giddens y Sutton recuerdan cuatro dimensiones de la exclusión social: además de pobreza o exclusión de una renta o unos recursos adecuados y de la exclusión del mercado de trabajo, también la exclusión de servicios y la exclusión de relaciones sociales (2013, p. 623).

Resumiendo, cuando no se trata de estrategia, sino de programa (hasta el punto de que incluso el tiempo de atención -y con él las necesidades- pueden venir predeterminados), cuando el objetivo es "facilitar" recursos, en el mejor de los casos, y no garantizar derechos, cuando el mayor esfuerzo se pone -desde una perspectiva homogeneizadora- en hacer "encajar" a la persona en el sistema "de ayuda", éste pasa a ser el único protagonista, y el agente de dicho proceso, y no las personas. Una "tecnología de disciplina" que permite controlar conductas, comportamientos, aptitudes, mejorar rendimiento, poner a la persona donde resulte más útil (Foucault, 1993, pp. 58-59). O donde menos estorbe.

\section{CONCLUSIONES}

Más allá de la falta de rigor metodológico, e incluso del enfoque que subyace, en el que la intervención con las mujeres víctimas-supervivientes de la violencia de género se aproxima mucho más a una dinámica de control -si no reclusión, y medicalización- que a su empoderamiento, y en el que el resto de personas para las que es referente ocupan un lugar residual, merece la pena señalar el carácter lesivo de un documento que opta por explicar a las/os profesionales cuestiones aparentemente susceptibles de ser olvidadas por éstas/os, tales como que se ha de lograr un "clima de empatía", "revisar el plan de actuación individualizada en función de nuevas necesidades o circunstancias" y "recabar de la usuaria el consentimiento informado para el tratamiento de sus datos", entre otras.

Desde la conversión en un mero saber funcional del corpus disciplinar implicado, y la intervención prácticamente reducida a gestión de recursos, batiburrillo, frangollo son algunos de los términos que desde aquí se considera que podrían utilizarse para definir lo que se muestra como una nebulosa informe. Inadecuadamente se denominan "pautas de intervención" a un conjunto desordenado de imprecisos, ambiguos, e incluso incoherentes eslóganes a modo de tormenta de ideas. Esto se materializa en un documento que se estima, cuanto menos, improvisado: queda lejos de ser un instrumento que garantice la calidad de la intervención y un "plan de actuación individualizado", y en el que, por el contrario, aunar, equiparar, yuxtaponer y/o combinar sistema, programa, fase, carácter, tipo, objetivo, fin, etcétera, aleatoriamente, es un leitmotiv, y a éste se unen alarmantes reduccionismos,

de alojamiento y manutención; menos aún asegura el acceso a un alojamiento digno; menos aún adecuado para una familia con posibles necesidades especiales derivadas de un proceso tan complejo y devastador como el de la violencia de género. En cualquier caso, no cabe duda de que hemos pasado, del welfare al workfare (ver Rodríguez y Díez, 2018) donde los derechos universales quedan supeditados al logro de cierto tipo de productividad, no cualquiera, exitosa, dentro del mercado laboral. 
Aparicio Martín, E. Propuesta de intervención impulsada por la delegación del gobierno de España para la violencia de género. Análisis de un documento de referencia para

profesionales

significativas omisiones y vejatorias reiteraciones, entre otro sinfín de cuestiones objetivamente minimizadas.

El documento no podría describirse como técnico, profesional. Menos aún puede considerarse que sirva "de referencia" para profesionales. En todo caso depende, claro está, de la imagen y el concepto que se tenga sobre el sector/sectores en el/los que podría ubicarse la intervención y las disciplinas y profesionales a las/os que se alude directa o indirectamente, o que podrían darse por aludidas/os. Este documento es un magnífico ejemplo de cómo un intento de regulación puede lograr el efecto contrario al que busca. Este documento no sólo no debiera definirse como técnico, sino que debiera entenderse como estrategia de desprofesionalización (curiosamente en un sector feminizado); no sólo no contribuye a una intervención de calidad, sino que la obstaculiza. La intervención social no puede frivolizarse, ni trasladarse una imagen simplificadora de su complejidad, y este documento así lo hace.

Los derechos reconvertidos en privilegios, las llamadas a la responsabilidad de las víctimas-supervivientes, y la constante apelación a mecanismos de control, nos llevan a un enfoque de sospecha, incluso punitivo (si no abiertamente por la situación que se afronta, sí por el riesgo que parece ser se presupone de mal uso de tiempo y recursos utilizados para la superación de ésta) es decir: re-victimización. No se considera impertinente, por tanto, concluir que lo único que trae consigo es un grave daño a las partes implicadas. Aunque justamente supone un magnífico exponente del estado de la cuestión.

\section{REFERENCIAS}

Aretio, A. (2018). El abordaje de la violencia de género desde el sistema sanitario en Tajahuerce, I. \& Ramírez, E. (Eds.), La intervención en violencia de género desde diversos ámbitos (pp. 69-88). Madrid: Dykinson.

Bauman, Z. (2009). El arte de la vida. Barcelona: PAIDÓS.

Bauman, Z. y Donskis, L. (2015). Ceguera moral. Barcelona: PAIDÓS.

Cuenca, J.; Sánchez, J. E. y Burbano, M. H. (2014). Intervención social: algunas pistas para su comprensión y para trazar otros horizontes posibles, en Grupo de Intervención y Responsabilidad Social, Intervención Social y el debate sobre 10 público: reflexiones conceptuales y casos locales (pp. 69-90). Cali: Universidad ICESI. Recuperado de https://repository.icesi.edu.co/biblioteca digital/bitstream/10906/77507/1/fajardo intervencion social 2014.pdf

Defensorías del Pueblo (octubre, 2018). Declaración de las Defensorías del Pueblo sobre la atención a mujeres y menores víctimas de violencia de género. XXXIII Jornadas de Coordinación de Defensores del Pueblo. Alicante: Autor. Recuperado de http://www.elsindic.com/wp-content/uploads/2018/10/declaracion-defensoriasviolencia-genero.pdf 
Aparicio Martín, E. Propuesta de intervención impulsada por la delegación del gobierno de España para la violencia de género. Análisis de un documento de referencia para

profesionales

Foucault, M. (1993). Las redes del poder. Buenos Aires: Almagesto.

Giddens, A. y Sutton, P. W. (2013). Sociología. Madrid: Alianza.

Giroux, H. (2015). Pedagogías disruptivas y el desafío de la Justicia Social bajo regímenes neoliberales, Revista Internacional de Educación para la Justicia Social (RIEJS), 4(2), 13-27. Recuperado de http://dx.doi.org/10.15366/riejs2015.4.2

Martín Rojo, L. (2003). El análisis crítico del discurso. Fronteras y exclusión social en los discursos, en Íñiguez, L. (Ed.), Análisis del discurso. Manual para las ciencias sociales (pp. 157-191). Barcelona: UOC.

Mateo, Y. y Bueno, M. J. (2018). La violencia de género desde el punto de vista de la psicología, en Tajahuerce, I. y Ramírez, E. (eds.), La intervención en violencia de género desde diversos ámbitos (pp. 55-68). Madrid: Dykinson.

Miranda, M. (2009). De la caridad a la ciencia. Pragmatismo, Interaccionismo simbólico y Trabajo Social. Zaragoza: Mira Editores.

ONU Mujeres (2015). Un marco de apoyo a la prevención de la violencia contra la mujer. Recuperado de

http://www.unwomen.org/es/digital-library/publications/2015/11/preventionframework

Saavedra, J. (2015). Cuatro argumentos sobre el concepto de intervención social, Cinta moebio, 53, 135-146.

Recuperado de http://www.moebio.uchile.cl/53/saavedra.html

Rodríguez, J. R. y Díez, E. J. (2018). Dispositivos biopolíticos de integración de la exclusión social: el negocio de la educación de los pobres, Revista Internacional de Educación para la Justicia Social (RIEJS), 7(2), 129-144. Recuperado de https://doi.org/10.15366/riejs2018.7.2.008

Van Dijk,T. (2002). El análisis crítico del discurso y el pensamiento social, Athenea digital, Revista de pensamiento e investigación social, 1, 18-24. Recuperado de https://atheneadigital.net/article/view/n1-van/22-pdf-es

Van Dijk, T. (2016). Análisis crítico del discurso, Revista Austral de Ciencias Sociales, 30, 203-222.

Recuperado de http://revistas.uach.cl/index.php/racs/article/view/871

Zamanillo, T. (2012). Las relaciones de poder en las profesiones de ayuda. Una cuestión ética de primer orden, AZARBE, Revista Internacional de Trabajo Social y Bienestar, 1, 157-170.

Recuperado de https://revistas.um.es/azarbe/article/view/151211 
Aparicio Martín, E. Propuesta de intervención impulsada por la delegación del gobierno de España para la violencia de género. Análisis de un documento de referencia para

profesionales

Zamanillo, T. y Martín, M. (2011). La responsabilidad política del trabajo social, Trabajo Social Global, 2(3), 97-115.

Recuperado de http://revistaseug.ugr.es/index.php/tsg/article/view/921

\section{AUTORA:}

\section{Encarnación Aparicio Martín}

Pedagoga social y maestra, se especializa en Detección e Intervención en Violencia de Género y en Igualdad de Oportunidades. Doctoranda y docente colaboradora de la Universidad Complutense de Madrid, cuenta con una amplia trayectoria en la Intervención Social, especialmente en la atención a población adulta. Centra sus investigaciones en el campo de la Pedagogía Social Crítica.

e.aparicio@ucm.es

Orcid ID: https://orcid.org/0000-0001-6155-630X

Google Scholar:

https://scholar.google.es/citations?user=QL182yIAAAAJ\&hl=es\&oi=ao

\section{ANEXO:}

Documento de estudio:

http://www.violenciagenero.igualdad.mpr.gob.es/profesionalesInvestigacion/asistenci aSocial/protocolos/pdf/Punto5PropuestaPAI.pdf 